\title{
Crystallographic Angles For
}

\author{
Magnesium, Zinc and Cadmium
}

\author{
by Edward I. Salkovitz
}

$\mathbf{T}$ $\mathrm{HE}$ determination of the orientation of metal single crystals and the studies of plastic deformation are greatly facilitated by the use of the stereographic projection. To draw a standard projection of the crystal it is necessary to have a tabulation of the angles between the various crystallographic planes of the particular crystal studied. Bozorth $^{1}$ has published such a table for the cubic system which can be found in many reference books; such convenient tabulation for the hexagonal metals, however, does not appear to be in the literature, although scores of workers have calculated these angles. Therefore, Table I of angles for magnesium, zinc, and cadmium has been compiled. Since the $c / a$

Table I. Angles Between the Crystallographic Planes in Close-Packed Hexagonal Crystals of Magnesium, Zinc, and Cadmium

\begin{tabular}{|c|c|c|c|c|c|c|c|}
\hline \multirow{2}{*}{$\begin{array}{c}\text { HKIL } \\
0001\end{array}$} & \multirow{2}{*}{$\frac{h k i l}{10 \overline{1} 8}$} & \multicolumn{2}{|c|}{$\begin{array}{c}\text { Magnesium } \\
(\mathrm{c} / a=1.6235)\end{array}$} & \multicolumn{2}{|c|}{$\begin{array}{c}\text { Zinc } \\
(c / a=1.8563)\end{array}$} & \multicolumn{2}{|c|}{$\begin{array}{c}\text { Cadmium } \\
(c / a=1.8859)\end{array}$} \\
\hline & & $13^{\circ}$ & $11.3^{\prime}$ & $15^{\circ}$ & $0.0^{\prime}$ & $15^{\circ}$ & $13.6^{\prime}$ \\
\hline & $10 \overline{1} 7$ & $14^{\circ}$ & $59.6^{\prime}$ & $17^{\circ}$ & $1.5^{\prime}$ & $17^{\circ}$ & $16.8^{\prime}$ \\
\hline & $10 \overline{1} 6$ & $17^{\circ}$ & $33.5^{\prime}$ & $19^{\circ}$ & $39.5^{\prime}$ & $19^{\circ}$ & $56.8^{\prime}$ \\
\hline & $10 \overline{15}$ & $20^{\circ}$ & $33.2^{\prime}$ & $23^{\circ}$ & $12.3^{\prime}$ & $23^{\circ}$ & $32.1^{\prime}$ \\
\hline & $10 \overline{14}$ & $25^{\circ}$ & $6.7^{\prime}$ & $28^{\circ}$ & $10.6^{\prime}$ & $28^{\circ}$ & $33.8^{\prime}$ \\
\hline & $20 \overline{2} 7$ & $28^{\circ}$ & $10.5^{\prime}$ & $31^{\circ}$ & $29.0^{\prime}$ & $31^{\circ}$ & 53.3' \\
\hline & $10 \overline{1} 3$ & $32^{\circ}$ & $0.1^{\prime}$ & $35^{\circ}$ & $32.7^{\prime}$ & $35^{\circ}$ & $58.5^{\prime}$ \\
\hline & $20 \overline{2} 5$ & $36^{\circ}$ & $51.1^{\prime}$ & $41^{\circ}$ & $24.6^{\prime}$ & $41^{\circ}$ & $3.4^{\prime}$ \\
\hline & $10 \overline{12}$ & $43^{\circ}$ & $8.9^{\prime}$ & $46^{\circ}$ & $59.0^{\prime}$ & $47^{\circ}$ & $26.1^{\prime}$ \\
\hline & $20 \overline{2} 3$ & $51^{\circ}$ & $20.0^{\prime}$ & $55^{\circ}$ & $1.0^{\prime}$ & $56^{\circ}$ & $12.8^{\prime}$ \\
\hline & $10 \overline{1} 1$ & $61^{\circ}$ & $55.6^{\prime}$ & $64^{\circ}$ & $59.5^{\prime}$ & $65^{\circ}$ & $20.1^{\prime}$ \\
\hline & $20 \overline{2} 1$ & $75^{\circ}$ & $4.0^{\prime}$ & $76^{\circ}$ & $52.2^{\prime}$ & $77^{\circ}$ & 4.1' \\
\hline & $10 \overline{1} 0$ & $90^{\circ}$ & $0.0^{\prime}$ & $90^{\circ}$ & $0.0^{\prime}$ & $90^{\circ}$ & $0.0^{\prime}$ \\
\hline & $21 \overline{3} 2$ & $68^{\circ}$ & $2.4^{\prime}$ & $70^{\circ}$ & $37.1^{\prime}$ & $70^{\circ}$ & $51.4^{\prime}$ \\
\hline & $21 \overline{3} 1$ & $78^{\circ}$ & $36.9^{\prime}$ & $79^{\circ}$ & $59.8^{\prime}$ & $80^{\circ}$ & $9.2^{\prime}$ \\
\hline & $21 \overline{3} 0$ & $90^{\circ}$ & $0.0^{\prime}$ & $90^{\circ}$ & $0.0^{\prime}$ & $90^{\circ}$ & $0.0^{\prime}$ \\
\hline & $11 \overline{2} 8$ & $22^{\circ}$ & 5.3 & $24^{\circ}$ & $53.7^{\prime}$ & $25^{\circ}$ & $14.5^{\prime}$ \\
\hline & $11 \overline{2} 6$ & $28^{\circ}$ & $25.3^{\prime}$ & $31^{\circ}$ & $44.9^{\prime}$ & $32^{\circ}$ & $9.3^{\prime}$ \\
\hline & $11 \overline{2} 4$ & $39^{\circ}$ & $4.2^{\prime}$ & $42^{\circ}$ & $51.7^{\prime}$ & $43^{\circ}$ & $19.1^{\prime}$ \\
\hline & $11 \overline{2} 2$ & $58^{\circ}$ & $22.2^{\prime}$ & $61^{\circ}$ & $41.3^{\prime}$ & $62^{\circ}$ & $3.9^{\prime}$ \\
\hline & $11 \overline{2} 0$ & $90^{\circ}$ & $0.0^{\prime}$ & $90^{\circ}$ & $0.0^{\prime}$ & $90^{\circ}$ & $0.0^{\prime}$ \\
\hline & $12 \overline{3} 2$ & $68^{\circ}$ & $2.4^{\prime}$ & $70^{\circ}$ & $37.1^{\prime}$ & $70^{\circ}$ & $51.4^{\prime}$ \\
\hline & $1 \overline{2} \overline{3} 1$ & $78^{\circ}$ & $36.1^{\prime}$ & $79^{\circ}$ & $59.6^{\prime}$ & $80^{\circ}$ & $9.2^{\prime}$ \\
\hline & $12 \overline{3} 0$ & $90^{\circ}$ & $0.0^{\prime}$ & $90^{\circ}$ & $0.0^{\prime}$ & $90^{\circ}$ & $0.0^{\prime}$ \\
\hline \multirow[t]{4}{*}{$10 \overrightarrow{10}$} & $21 \overrightarrow{30}$ & $19^{\circ}$ & $6.4^{\prime}$ & $19^{\circ}$ & $6.4^{\prime}$ & $19^{\circ}$ & $6.4^{\prime}$ \\
\hline & $11 \overline{2} 0$ & $30^{\circ}$ & $0.0^{\prime}$ & $30^{\circ}$ & $0.0^{\prime}$ & $30^{\circ}$ & $0.0^{\prime}$ \\
\hline & $12 \overline{30}$ & $40^{\circ}$ & $53.6^{\prime}$ & $40^{\circ}$ & $53.6^{\prime}$ & $40^{\circ}$ & $53.6^{\prime}$ \\
\hline & $0 \overline{110}$ & $60^{\circ}$ & $0.0^{\prime}$ & $60^{\circ}$ & $0.0^{\prime}$ & $60^{\circ}$ & $0.0^{\prime}$ \\
\hline
\end{tabular}

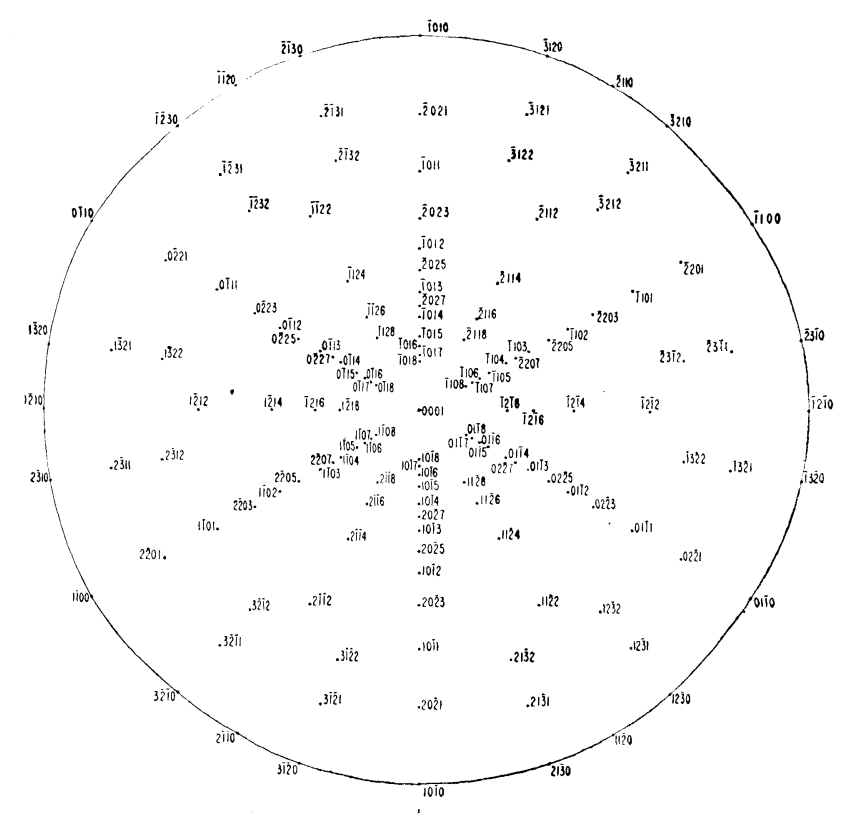

Fig. 1-Standard (0001) projection for zinc hexagonal $c / a=1.856$.

ratio is different for each of these metals, three separate calculations are necessary.

The angles $\phi$ between (HKIL) and (hkil) were calculated by means of the formula:

$$
\cos \phi=\frac{H h+K k+\frac{1}{2}(H k+h K)+\frac{3}{4} \frac{a^{2}}{c^{2}} L l}{\left[\left(H^{2}+K^{2}+H K+\frac{3 a^{2}}{4 c^{2}} L^{2}\right)\left(h^{2}+k^{2}+h k+\frac{3 a^{2}}{4 c^{2}} l^{2}\right]^{1 / 2}\right.}
$$

The $c / a$ ratios were calculated from data given in the 1948 edition of Metals Handbook. Fig. 1 is a typical (0001) standard projection of zinc. For all practical purposes, it serves for cadmium as well, as can be seen from the table. For magnesium, however, it is necessary to construct another net.

${ }^{1}$ R. M. Bozorth: Physical Review. (1925) 26, 390.

EDWARD I. SALKOVITZ is on the staff of the U. S. Naval Research Laboratory of the Office of Naval Research, Washington, D. C.

TN 50 E. Manuscript, Oct. 10, 1950. Part of these calculations were made at Carnegie Institute of Technology. 